\title{
Motor speech and motor limb status in athletes following a concussion
}

\author{
Anthony P. Salvatore' ${ }^{1}$ Michael P. Cannito ${ }^{1}$, Jessica Hewitt ${ }^{2}$, Lindsay D. Dolan², George King ${ }^{3}$, Heather E. Brassil² \\ 'University of Louisiana-Lafayette, Louisiana: ${ }^{2}$ University of Texas at El Paso, El Paso; ${ }^{3}$ Texas Woman's University, Denton, USA
}

Purpose: There is limited research on the effects of an acute concussion on motor speech production in student-athletes. This study investigated the effects of a concussion on (1) speech alternating motion rates and (2) finger repetition task.

Methods: Eighteen individuals with a concussion and 18 individuals in a healthy comparison (HC) group, matched for age and years of education served as participants. Oral diadochokinetic sequential motion rates (SMR) and alternating motion rates (AMR) were measured using Kay Elemetrics Computerized Speech Lab, model 4500. A finger repetition task was also evaluated.

Results: Independent-samples t-tests revealed statistically significant differences in mean syllable repetition rate between groups: SMRs $(p<0.001)$, AMR /puh/ $(p<0.001)$, AMR /tuh/ ( $p<0.001)$, and AMR /kuh/ $(p<0.001)$. Also, a statistically significant difference was found for the finger repetition task $(p<0.05)$.

Conclusions: Participants with a concussion produced SMR and AMR speech-like tasks and finger repetition tasks significantly slower than a matched healthy control group.

Keywords: Motor speech, Concussion, AMR

\section{INTRODUCTION}

Motor speech disorders have a significant impact on an individual's quality of life, social interaction, employment, academic performance, and self-esteem. The prevalence of motor speech disorders following a sports-related concussion (SRC) is limited, specifically in the case of severe concussions, repeated concussions, an occurrence of intracranial bleeding, and brainstem and peripheral nerve damage that may occur secondary to an SRC [1]. What data does exist is limited to a small number of cases reported as part of a larger sample of severe TBI, and across several different sports activities. The need for the identification of motor speech dysfunction in a well-defined group of student-athletes will make a significant contribution to our understanding of the consequences of SRC on the cognitive-communication behavior of student-athletes.

According to the Centers for Disease Control and Prevention [2], 138 people die every day in the United States, from traumatic brain injuries (TBI). The yearly incidence of TBI is estimated to be between 1.6 million and 3 million for recreational sports [2]. A large percentage of these victims are within the pediatric range, 5-18 years old, which accounts for the majority of sports-related concussions [3]. Gessel et al. [4] reported that the majority of concussions, at the high school level, result from participation in

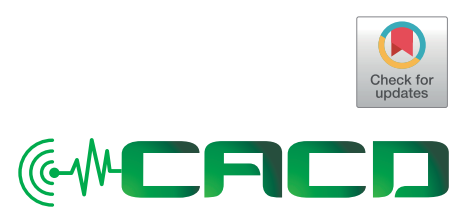

Received: October 30, 2019 Revision: December 16, 2019 Accepted: December 18, 2019

Correspondence: Anthony P. Salvatore

Department of Communicative Disorders, University of LouisianaLafayette, P.O. Box 43170, Lafayette, LA 70504-3170, USA

Tel: $+915-861-4427$

Fax: +222-222-2222

E-mail: asalvatore@louisiana.edu

(C) 2019 The Korean Association of SpeechLanguage Pathologists

This is an Open Access article distributed under the terms of the Creative Commons Attribution NonCommercial License (http://creativecommons.org/ licenses/by-nc/4.0/) which permits unrestricted noncommercial use, distribution, and reproduction in any medium, provided the original work is properly cited. 
the following sports: football- $40 \%$, girls' soccer- $22 \%$, boys' soccer- $15 \%$, and girls' basketball $10 \%$. However, the true incidence of sport-related concussion (SRC) is unknown since many concussions go unreported [5]. Many individuals do not realize they have suffered a concussion or mild traumatic brain injury (mTBI). Understanding the consequences of a concussion can be challenging. A concussion may not be immediately noticeable and can go undetected, because there is no structural damage or obvious physical abnormality immediately after a concussion, [6] athletes and parents, all too often, fail to understand its impact, severity, and complexity.

A concussion is a subset of TBI, classified as an mTBI, and defined as a "complex pathophysiological process affecting the brain, induced by biomechanical forces" [7]. An mTBI is produced by a biomechanical force created by a direct blow to the head, neck, or body with significant force to put the brain in motion [7]. As a result of this biomechanical force, the brain experiences linear, rotational, and angular acceleration that results in a broad spectrum of severity, contingent upon the site of the impact of the traumatizing force and direction of the force [8]. Following an mTBI, the brain goes into a state of temporal neuronal dysfunction, an event characterized by anionic shift, altered metabolism, impaired connectivity, or changes in neural signaling [9]. Other variables, such as a history of concussion and length of retrograde or anterograde amnesia, also play a factor in the severity of a mild TBI/concussion.

Given that a concussion is a closed head injury, producing cortically and subcortical dysfunction affecting the entire brain, multiple neurological functions may be impacted adversely. Different brain regions and white matter fiber tracts are affected. However, there remains a lack of understanding and uncertainty as to the impact of an SRC on motor speech functioning [1]. Motor speech disorders, according to Duffy [10], result from neurologic impairments that consequently affect the planning, programming, control, or execution of speech. Dysarthria is a group of neurologic speech disorders that may reflect abnormalities in all or some of the following speech parameters: strength, speed, range, steadiness, tone, or accuracy of movements required for breathing, phonation, resonation, articulation, or prosody. Motor speech dysarthria is not an uncommon outcome following a TBI [11-20], however, there are limited reports exploring motor speech dysarthria as an outcome of SRC [1].

Motor speech parameters are assessed commonly via measures of oral diadochokinesis (DDK) speech productions.
These measures are sensitive to mild neuromuscular impairment of the lips, tongue, and vellum [20]. DDK tasks include both: alternating motion rates (AMR) and sequential motion rates (SMR) of the speech musculature. These two measures compare the speech motor demands of a single sound repetition task versus a syllable sequence repetition task [21]. DDK tasks are non-lexical tasks that assess articulatory and speech motor planning. Repetitions of these non-meaningful/lexical syllables are likely to yield a measure of speech motor ability less contaminated by linguistic factors than a real word or non-word repetition [21].

Alternating motion rates (AMR) are useful tasks in judging speed and regularity of reciprocal jaw, lip, and anterior/posterior tongue movements, while also detecting articulatory precision, adequacy of velopharyngeal closure and respiratory and phonation support [10]. AMRs are characterized by the steady repetition of a monosyllable such as, /puh, puh, puh.../ without any variance in syllable type; this task may also include monosyllables: /tuh/ and /kuh/ [22]. Sequential motion rates (SMRs) are useful in detecting deficits of sequential movements from one artciulator to another [10]. The production of SMRs is accomplished by repeating three different syllables, put together, continuously: /puhtuhkuh, puhtuhkuh, puhtuhkuh/ [21].

Motor speech production deficits, post-TBI, are characterized by impaired speech rates and reduced speech intelligibility [1]. Cahill and colleagues1 [7] reported significant differences in intellibilility and speech rates in children with and without motor speech dysarthria following TBI compared to matched control group. Sixteen children with a TBI and motor speech dysarthria and eight children with TBI and no motor speech dysarthria were compared to helathy control group of matched children. The children with TBI \& speech dysarthria were statistically less intellibible than the control group of children. However, there are no current studies reporting speech rate measures following an SRC in adolescents and adults. Furthermore, since SRCs represent a mild form of TBI, it is plausible that the consequences may result in generalized motor execution slowness or bradykinesia [23]. De Beaumont et al. [23] measured cognitive and motor functions in former university athletes (mean age $=60$ years), $15-25$ years postSRC, and a control group (mean age $=59$ years) of 21 athletes with no history of SRC. They concluded that relative to controls, the concussed group exhibited cognitive and motor system alterations.

The purpose of the current study is to examine the impact 
of an SRC on motor speech and motor limb movements, in adolescents and adults, during the acute phase of an SRC. The following speech parameters were assessed; AMR and SMR tasks, in addition to a finger repetition task. The two objectives of this study were to determine if concussed athlete's DDK performance was impacted adversely by a sports-related concussion and secondly to determine if there are statistical correlations between AMR and SMR tasks and finger repetition task.

\section{METHODS}

The current study was reviewed and approved by the Institutional Review Board (IRB) \#552011-2 University of Texas at El Paso. Signed informed consent was obtained from all participants, including parents if participants were under the age of 18.

\section{Participants}

Inclusion criteria for the healthy control (HC) and SRC groups were the following: (a) age range 13-23; (b) no hearing loss; (c) no history of drug or alcolhol abuse; (d) no previous history of psychiatric illnesses; (e) no previous history of a learning disability; (f) no history of seizures, brain tumors, et.: and (Eng- lish as a domiant language. Also, the SRC group members all had a current diagnosis of a concussion determined by a physician and trainer. One of the athletes in the SRC group suffered a whiplash injury but was included given the evidence that the biomechanical mechanism of the injury is similar to that of a concussion. Furthermore, this participant's performance was similar to that of the other athletes in the SRC group [24]. The HC and SRC groups, each consisted of 18 individuals, matched for age and education to the SRC group. The HC group did not report experiencing a concussion 12 months prior to testing. Table 1 displays the demographic information for the individual participants in the SRC group, and Table 2 shows the information for the individual participants in the HC group. Table 3 summarizes the demographic information for both groups.

All assessments were carried out within 1-33 days of their concussion. Of the 18 athletes who sustained a concussion, seventeen were sport-related concussions at the junior high, high school, or collegiate level. Participants in the SRC group were recruited from the files of the University of Texas at El Paso's Concussion Management Clinic. Individuals diagnosed with a concussion by an athletic trainer and/or a physician were referred to the clinic. Upon completion of the protocol, participants received a $\$ 25$ gift card.

Table 1. Demographic information for the sports-related concussion group

\begin{tabular}{|c|c|c|c|c|c|}
\hline Subject & Gender & Age & Concussion type and level & History of concussion & Days post injury \\
\hline 201 & $\mathrm{~F}$ & 23 & Whiplash-MVA & 0 & 3 days \\
\hline 202 & M & 13 & Sport: Football (Middle School) & 3 & 7 days \\
\hline 203 & $\mathrm{~F}$ & 21 & Sport: Cheerleading (Collegiate) & 0 & 3 days \\
\hline 204 & M & 18 & Sport: Ice Hockey (Semi-Professional) & 2 & 1 day \\
\hline 205 & $\mathrm{~F}$ & 18 & Sport: Cheerleading (Collegiate) & 2 & 2 days \\
\hline 206 & M & 20 & Sport: Cheerleading (Collegiate) & 3 & 4 days \\
\hline 207 & M & 17 & Sport: Football (High School) & 1 & 4 days \\
\hline 208 & M & 22 & Sport: Football (Collegiate) & 0 & 7 days \\
\hline 209 & M & 17 & Sport: Football (High School) & 3 & 32 days \\
\hline 210 & M & 16 & Sport: Football (High School) & 0 & 33 days \\
\hline 211 & M & 22 & Sport: Football (Collegiate) & 1 & 2 days \\
\hline 212 & $\mathrm{~F}$ & 18 & Sport: Basketball (Collegiate) & 0 & 1 day \\
\hline 213 & $\mathrm{~F}$ & 19 & Sport: Basketball (Collegiate) & 1 & 6 hours \\
\hline 214 & M & 18 & Sport: Ice Hockey (Semi-Professional) & 3 & 4 days \\
\hline 215 & M & 19 & Sport: Ice Hockey (Semi-Professional) & 3 & 12 days \\
\hline 216 & $\mathrm{~F}$ & 19 & Sport: Cheerleading (Collegiate) & 0 & 6 days \\
\hline 217 & M & 19 & Sport: Ice Hockey (Semi-Professional) & 0 & 3 days \\
\hline 218 & $\mathrm{~F}$ & 19 & Sport: Dance (Collegiate) & 0 & 2 day \\
\hline
\end{tabular}


Table 2. Demographic information for the healthy control group

\begin{tabular}{lcclc}
\hline Subject & Gender & Age & \multicolumn{1}{c}{ Sport } & Medical history \\
\hline 101 & F & 21 & Non-Athlete & None \\
102 & F & 23 & Mountain Biking & None \\
103 & F & 21 & Track \& field & None \\
104 & M & 20 & Non-Athlete & None \\
105 & F & 17 & Basketball & None \\
106 & M & 23 & Non-Athlete & None \\
107 & F & 20 & Non-Athlete & None \\
108 & M & 18 & Non-Athlete & None \\
109 & F & 22 & Non-Athlete & None \\
110 & F & 21 & Non-Athlete & None \\
111 & M & 23 & Baseball & None \\
112 & F & 16 & Non-Athlete & None \\
113 & F & 18 & Non-Athlete & None \\
114 & $\mathrm{M}$ & 13 & Soccer & None \\
115 & $\mathrm{M}$ & 22 & Non-Athlete & None \\
116 & $\mathrm{M}$ & 23 & Non-Athlete & None \\
117 & $\mathrm{~F}$ & 18 & Non-Athlete & None \\
118 & $\mathrm{~F}$ & 15 & Track \& field & None \\
\hline & & & &
\end{tabular}

Participants in the HC group were recruited from various academic programs in the Department of Rehabilitation Sciences at the University of Texas at El Paso. Adolescents were mainly younger siblings of university students. Upon completion of the protocol, these participants received a $\$ 10$ gift card.

\section{Setting}

The study was carried out in a clinical setting for testing and to meet with all the participants, sign consent forms, orally explain the testing procedures, fill out initial participant medical history form, and to disseminate treatment recommendations for participants in the SRC group. Hearing screenings and recording of speech and motor movements were carried out in a sound-proof booth.

\section{Procedure}

Consent forms and explanation of testing procedures took place before any testing began. The process began with the administration of (a) medical history questionnaire and (b) hearing screening test. If any of the inclusion criteria were not met, participants were automatically dismissed. The SRC group and the HC group both underwent a standard proto$\mathrm{col} /$ battery. The test battery assessed auditory comprehen-
Table 3. Summary of demographic information for both groups

\begin{tabular}{lccc}
\hline Characteristics & Control group & SRC group & Significance \\
\hline Age & $19.56(2.94)$ & $18.83(2.43)$ & NS \\
Years of Ed. & $12.83(2.85)$ & $12.17(2.31)$ & NS \\
Hours of sleep & $7.36(1.88)$ & $7.74(2.46)$ & NS \\
Gender & 7 Males, & 11 Males, & \\
& 11 Females & 7 Females & \\
Handedness: R (right), & $\mathrm{R}=18 ; \mathrm{L}=0 ;$ & $\mathrm{R}=15 ; \mathrm{L}=2 ;$ & \\
$\mathrm{L}$ (left) A (ambidextrous) & $\mathrm{A}=0$ & $\mathrm{~A}=1$ & \\
Adolescents (13-17 yr) & 4 & 4 & \\
Adults (18-24 yr) & 14 & 14 &
\end{tabular}

Standard deviations are represented in parenthesis. NS=not statistically significant.

sion, short-term memory, word fluency, simple learning task, copying drawlings, balance, and symptom questionnaire.

Upon confirmation of the participant's eligibility, the testing protocol was administered to the SRC group in addition to the Oral Diadochokinesis (DDK) tasks and the motor limb task. The administration of the entire protocol was accomplished during one clinical visit. The protocol took one and a half to two hours in duration to complete. Two levels of randomization were utilized in the administration of the protocol to account for test-fatigue. The standard post-concussion protocol tasks were administered in the same order to each SRC individual; however, the DDK, and motor limb task were administered either at the beginning or the end of the standard protocol. An additional level of randomization was carried out within the DDK tasks. Four conditions were created. Two conditions were administered before the standard protocol: eliciting SMRs first and then AMRs or AMRs first and then SMRs; or two conditions at the end of the standard protocol: eliciting SMRs first and then AMRs or AMRs first and then SMRs. The motor limb task was always administered following the speech tasks.

\section{Speech Tasks}

\section{Oral Diadochokinesis Tasks}

The Computerized Speech Lab (CSL) model 4500 by Kay Elemetrics [25], with a sampling Hz of 11025 [26], was used to record the DDK tasks for both groups, which were carried out inside a sound-treated booth. Participants sat across from the examiner. A condenser microphone (model C420) was positioned approximately one inch from the center of the mouth of the participant. The examiner read aloud the standardized instructions and modeled the desired DDK responses. Each 
participant was allowed to practice the tasks but not exceeding three trials. This practice opportunity assured that every participant understood the tasks before proceeding. For individuals producing inaccurate DDK productions, such as a repetition of a consonant in either AMRs or SMRs, they were asked to repeat the sequence until an accurate production was obtained.

\section{Alternating Motion Rates}

To produce the alternating motion rates (AMR), participants were instructed to take a breath and repeat the monosyllables /puh/, as fast and as accurately as possible, then /tuh/, and then /kuh/. After the production of 12 repetitions of each syllable, the participant was asked to stop. Participants did not have to complete any of these tasks under a time constraint. Of the 12 syllable productions, the first and last syllables were eliminated from the analysis. The mean syllable rate and total mean rate for the remaining ten repetitions were analyzed using the CSL. The same process was repeated for the /tuh/, /puh/ and /kuh/ syllables.

\section{Sequential Motion Rate}

Each participant, to produce the sequential motion rate (SMR), was instructed to take a breath and produce the trisyllable: /puhtuhkuh/ as quickly and as accurately as possible. Twelve repetitions of the trisyllable sequences were recorded, and then the participant was asked to stop. If any misarticulation was noted during the recording, the participant was asked to produce the sequence again. Participants did not have to complete any of these tasks under a time constraint. Of the 12 syllable productions, the first and last syllables were eliminated from the analysis.

\section{Motor Limb Task}

\section{Finger Repetition Task}

The finger repetition task was administered to each participant group. Each participant was seated in a chair across from the examiner. A Mac Book Pro computer was used to videorecord the participant's productions for scoring and reliability purposes. It was placed on top of a computer table, next to the examiner facing the participant directly and ready to begin video recording. The examiner read the standardized instructions and then directed the participant to perform the finger repetition task. The task required the participant to alternate their fingers, beginning with a contact of the thumb to index finger followed by the thumb to the middle finger and finally thumb to ring finger, in a sequential motion. Each participant was asked to focus on speed and accuracy. With their dominant hand, each participant was allowed to practice two productions of the finger repetition task to ensure the participant understood the task before commencing. When ready to begin, the examiner began the video recording using the Photo Booth camera, version 7.0. A total of 12 finger repetitions were recorded. The first and last finger repetitions were eliminated from the analysis; only ten-finger repetitions were later timed using video playback.

\section{Acoustic Analysis}

The CSLwas used to analyze and calculate the DDK durations for ten productions of SMR and AMR. Thus, there were a fixed number of syllable occurrences elicited for each DDK task. The mean duration for each production was measured, and a mean duration per syllable calculated. The mean rate of repetition, the reciprocal of mean duration was calculated and reported.

For each analysis, a combination of the waveform and spectrogram displays were simultaneously aligned on the CSL screen, sized to the same window size, and the cursors were linked. The waveform display was the primary source of analysis supplemented with the auditory along with an audio perception of the examiner. In analyzing the onset of the initial syllable and the offset of the final syllable consistently, a 0.3-second window expansion was utilized both at the beginning and again at the end, to measure all of the DDK sequences accurately. The beginning cursor was placed manually where the waveform came off of the baseline, and the end cursor was placed where the waveform flatlined back to the baseline. However, if there was any obscurity in identifying the onset and/or offset of a syllable, the spectrogram display was utilized as a secondary form of analysis to determine the onset or offset of the syllable. Only ten productions were measured for each DDK task. Measurements began with the 2nd production and continued through the 11th production.

For the SMR task, the cursors were placed on the onset of the second /puh/ syllable and at the end of the offset of the final /kuh/ syllable. Again a 0.3-second window expansion was used to measure the onset and offset of the entire sequence. Once the beginning and end cursors were placed, the CSL calculated the total time for all ten /puhtuhkuh/ tri-syllable sequences with gap durations in seconds. Additionally, each /puhtuhkuh/ sequence was measured one at a time, using a 0.8 -second window expansion to measure the onset and off- 
set of each tri-syllable sequence for the SRC group and a .05 second window expansion was used for the control group. Cursors were placed at the beginning and end of each /puhtuhkuh/ sequence, and the CSL calculated the total duration time for each tri-syllable sequence.

In the AMR task, the cursors were placed manually on the onset of the 2nd production of the /puh/ syllable and at the offset of the final, 11th production, of the/puh/ syllable. A 0.3-second window expansion was used to measure the onset and offset of the entire sequence. Cursors were manually placed at the onset and offset of each /puh/ syllable to obtain the syllable duration time, with intersyllable gap durations. For each analysis, one to three /puh/ waveforms were analyzed at a time, using a 0.5-second window expansion for the SRC group and a 0.3-second window expansion for the HC group. The same analysis procedures were repeated to obtain the measurements of the other two syllables: /tuh/ and /kuh/. The mean syllable rate was calculated for comparison between the groups.

\section{Finger Repettion Task Analysis}

In the finger repetition task, a visual analysis using QuickTime player, version 10.4, was used. The examiner opened the participant recorded video and once opened selected the edit option, and then trim. This option allowed the examiner to view an enlarged screen of the video, while also providing small trimmings of the same video at the bottom with a cursor and time in seconds. As the examiner moved the cursor at the bottom along with the video trimmings, the finger repetitions were shown in the enlarged screen in slow motion. This allowed the examiner to accurately calculate the duration time for the finger repetitions using the time provided by the program. Only ten of the 12 finger repetition sequences were analyzed. Initial time began with the contact of the pointer finger and thumb of the second finger-sequence. The timing ended with the final contact of the ring finger and thumb of the 11th finger-sequence. Again the reciprocal rate was calculated for the recorded duration for the ten productions.

\section{Data Analysis}

A Shapiro-Wilk test of normality was calculated to determine if the groups were distributed normally. An independentsamples $t$-test ( $\alpha=0.05$ ) was used to examine group differences since this test bypasses the assumption of a normal distribution [26]. The $p$-value was not adjusted for multiple serial $t$-tests. Because the groups lacked a normal distribution, a non-parametric test: Mann-Whitney-U was calculated for all tasks.

The first research question asked if there were differences in DDK mean syllable rate between the groups. The independent variables were the SRC group and the H.C. group. The dependent variables were the DDK repetition rates. The second research question asked if there were differences in the mean repetition rate for the motor limb task between the groups. The independent variables were the SRC and H.C. groups, and the dependent variable was the finger repetition rate. Finally, a Spearman correlation coefficient was used to examine the relationship between speech and motor limb repetition rates.

\section{RESULTS}

\section{Reliability}

Standardized instructions were created and administered before each motor speech and motor limb task to ensure consistency across participants. An Intraclass Correlation Coefficient (ICC), one-way randomization was used to index all reliability measures.

Twenty-five percent of the DDK tasks in the SRC and the H.C. group were randomly re-measured by the third author. Table 4 shows the results of an ICC, one-way random, intrarater agreement for both groups.

Eighty percent of the finger repetition task, for the HC group and SRC group, were randomly selected to be re-measured from video recordings of each subject's performance. The finger repetition task was re-analyzed using the trim option in the QuickTime player, version 10.4. An ICC showed an interrater agreement of 0.912 for the HC group and 0.954 for the SRC group.

\section{Oral Diadochokinesis: Mean Syllable Repetition Rate}

The first research question sought to examine the differences in DDK mean syllable repetition rate between the SRC group and the HC group. Results showed smaller DDK mean sylla-

Table 4. Reliability measures for DDK tasks between both groups

\begin{tabular}{lll}
\hline DDK TASKS & SRC & HC \\
\hline SMR With GAP & 0.996 & 0.999 \\
PUH With GAP & 1.00 & 1.00 \\
TUH With GAP & 1.00 & 1.00 \\
KUH With GAP & 1.00 & 1.00 \\
\hline
\end{tabular}


ble repetition rate in the SRC group in comparison to the $\mathrm{HC}$ group. An independent-samples $t$-test revealed a statistically significant difference in DDK mean syllable repetition rate across groups: The SMR mean tri-syllable duration for the SRC group was 482 milliseconds \pm 80.17 or 2.07 mean repetition rate per sec. in comparison to 341 milliseconds \pm 34.93 or 2.93 mean repetition rate per sec. for the $\mathrm{HC}$ group.

The AMR mean syllable duration, in milliseconds, for each syllable, were the following: /puh/- 146 milliseconds \pm 27.32 or 6.84 mean repetition rate per sec. exhibited for the SRC group in comparison to 103 milliseconds \pm 14.25 or 9.70 mean repetition rate per sec. for the HC group; /tuh/- 183 milliseconds \pm 51.11 or 5.46 mean repetition rate per sec. for the SRC group in comparison to 119 milliseconds \pm 20.79 or 8.40 mean repetition rate per sec. for the HC group; /kuh/- 189 milliseconds \pm 46.55 or 5.29 mean repetition rate per sec. in the SRC group in comparison to 125 milliseconds \pm 22.33 or 8.00 mean repetition rate per sec. for the $\mathrm{HC}$ group.

Additional analysis demonstrated an overall smaller mean repetition rate across all DDK tasks, with and without intersyllable gap durations, in the SRC group in comparison to the HC group.

\section{Finger Repetition Task Between Groups}

An independent-sample $t$-test was calculated to answer the second research question; was there a significant difference in finger-repetition task between the groups. A statistically significant difference was found, between groups, in the finger repetition task $[t(34)=-2.124, p<0.05]$. The total mean finger repetition rate for the SRC group was 12 repetitions per sec. minute \pm 0.60 , and the total mean repetition rate for the HC group was 16 repetitions per sec. minute \pm 0.625 . A Spearman rho correlation coefficient was calculated for the relationship between all DDK tasks and finger repetition task across groups. A fragile relationship, that is not significant, was found between groups across DDK tasks and finger repetition task.

\section{DISCUSSION}

The current study investigated DDK tasks in the participants to determine if an acute SRC adversely impacts motor speech and finger repetition rate.

\section{DDK Tasks}

In the DDK task, it was hypothesized that DDK mean syllable repetition rates would be slower across SMRs and AMRs for the SRC group when compared to a HC group. The experimental findings support the hypothesis. The SRC group produced fewer SMRs per second than the HC group. These findings are consistent with studies that indicate that individuals post-TBI produce significantly fewer AMR rates when compared to control groups $[15,16,18,20]$.

Both Wang et al., $[15,16]$ studies measured AMR syllable duration times, the reciprocal of rate, as well as the inter-syllable gap durations. They report a simultaneous increase in syllable duration and inter-syllable gap duration. However, the findings of the current study did not show such a pattern. Although the SRC group displayed slightly longer mean intersyllable gap duration than the HC group, there was no statistically significant difference found. This finding in the current study indicates that the significant difference found in DDK repetition rate was a result of increased syllable length, rather than inter-syllable gap durations and syllable length.

Additionally, the Ziegler [18] study noted a pattern in the AMRs for almost all groups, made up of individuals with a TBI and individuals with other disorders: apraxia of speech, ataxic cerebellar disorders, cerebrovascular accident, closed head injury, and other non-specified disorders. This study also reports /puh/ as the fastest syllable produced, followed by the /tuh/ syllable and then the / kuh/ syllable. In the current study, the same pattern was exhibited in the SRC group and in the HC group.

The experimental findings for the DDK tasks are clinically significant because slowness was exhibited equally across AMRs and SMRs suggesting a motor speech disorder: dysarthria rather than apraxia of speech [1]. However, at this time, it is not clear that these individuals were experiencing dysarthria, but rather a motor slowing of the articulators. A more thorough speech analysis combined with the acoustic analysis is needed to define whether it is dysarthria that these individuals were experiencing post-SRC.

\section{Finger Repetition Task}

The current results do not validate the hypothesis that generalized motor system slowing can explain the slowed speech tasks and slowed finger repetition task for the SRC group compared to the HC group. A Spearman correlation coefficient showed no significant correlation existed between the mean DDK repetition rates and the finger repetition task, across groups. This correlation was of interest because of the anatomical similarities between the finger repetition sequence and the SMR task. The SMR task required the individ- 
ual to articulate, using their tongue in a specific sequence: from lips to the tongue tip, and then the tongue dorsum when producing the /puhtuhkuh/ sequence [18]. Likewise, the finger repetition task required the individual to move their first three fingers (index, middle, and ring) in a repetitive sequence as well. The lack of correlation may be explained based upon the following; each of these motor activities; motor speech, and finger repetition are mapped at different cortical locations, the finger movements for the dominant hand are mapped unilaterally versus the speech tasks which are bilaterally mapped.

\section{Limitations}

This study has some limitations which may warrant further investigations. For example, perceptually five of the participants in the SRC group produced slower speech rates, misarticulations, and monopitch voice characteristics in both SMRs and AMRs tasks. Further exploration comparing the DDK mean syllable rate and/or total duration time, and speech rate tasks is needed.

Additional investigations would be beneficial to determine if clinicians can distinguish the differences across these speech and voice characteristics between concussed and non-concussed athletes to delineate further speech characteristics consistently displayed by individuals with a concussion.

Concussion management protocols should consider including DDK tasks because these tasks may yield additional signs associated with the adverse impact of a concussion. DDK tasks can also highlight speech dysfunctions that cannot otherwise be masked by the athlete, or may not be immediately evident in neurocognitive testing.

Future studies should consider using non-concussed athletes at a similar level of competition for the control group. The HC group is primarily composed of university students, most of whom are non-athletes. Additionally, a small sample size, for both groups, continues to be a limitation. Larger sample size will aid in obtaining normal distribution across groups and may further increase statistical significance. Finally, due to the limited number of athletes in the SRC group, statistical analysis could not be calculated to account for fatigue or adolescent versus adult comparisons. Increasing the sample size for both groups will aid in controlling for these effects.

Finally, while the DDK task requires bilateral activation, the finger repetition task assessed in this study only tested the dominant hand finger movement. In the future, simultaneously assessing right and left hand fingers may prove interesting.

\section{CONCLUSION}

The experimental findings of the current study are promising. One of the most robust attributes of DDK tasks is their usefulness in detecting motor speech disorders by highlighting underlying motor speech impairments [21]. In this study, DDKs were analyzed acoustically, which allowed for the precise measurement of the mean syllable repetitions between the groups. Determining the impact of a concussion on motor speech and the frequent clinical situation of an athlete underreporting their symptoms can be addressed objectively using the acoustic analysis of DDK. For example, one of the adolescent athletes reported no symptoms at the time of testing; however, his DDK times revealed mild impairments averaging one of the longest DDK tasks durations within the SRC group. This observation suggests that DDK tasks can be a valuable tool in the clinical setting to determine the presence or absence of cortical/subcortical dysfunction of motor speech.

The experimental findings show that concussed athletes exhibit a motor slowing of articulators in comparison to control subjects. Cannito [1] delineates the process speech-language pathologists should follow when encountered by cranial damage, following an SRC, which may lead to a motor speech dysarthria. Futhermore, he explains that if there is evidence of cranial nerve involvement, individuals should be given a comprehensive motor speech evaluation that includes: a case history, speech mechanism examination, formal perceptual speech assessment, standardized dysarthria testing, and examination of non-speech motor behaviors.

Despite the improvements in concussion rules and regulations, the outcome and consequences of an SRC continue to weigh heavily on athletes. Parents, coaches, trainers, teachers, administrators, speech-language pathologists, and physicians must all work together to not only provide individualized care but also to ultimately become the athlete's fiercest advocates in caring for them as they develop.

\section{ACKNOWLEDGMENTS}

Portions of this work was submitted in partial fulfillment of the requirements for a M.S. degree in Speech-Language Pathology, University of Texas at El Paso by Ms. Dolan and Ms. Hewitt. Dr. Salvatore served as thesis chair for both of these individuals. 


\section{REFERENCES}

1. Cannito MP. Clinical assessment of motor speech disorders in adults with concussion. Seminars in Speech and Language. 2014; 35:221-233.

2. Centers for Disease Control and Prevention. Injury prevention and control: traumatic brain injury-TBI data and statistics. 2010, Retrieved from http://www.cdc.gov/ traumaticbraininjury/data/ index.html

3. Halstead ME, Walter KD. Clinical report-sport-related concussion in children and adolescents. American Academy of Pediatrics. 2010;126:597-615

4. Gessel LM, Fields SK, Collins CL, Dick RW, Comstock D. Concussions among United States high school and collegiate athletes. Journal of Athletic Training. 2007;42:495-503.

5. Grady MF. Concussion in the Adolescent Athlete. Curr Probl Pediatric Adolescent Health Care, 2010;40:154-169.

6. McCrea MA. Mild traumatic brain injury and post-concussion syndrome. Oxford, NY: Oxford University Press 2008.

7. McCrory P Meeuwisse WH Aubry M Cantu RC Dvorak J Echemendia RJ Engebretsen L et al. Consensus statement on concussion sport: the 4th international conference on concussion in sport, Zurich, November 2012. Journal of Athletic Training. 2013;48:554575 .

8. Kilbourne M Kuehn R Tosun C Caridi J Keledijan K Bochicchio G Scalea T Gerzanich V Simard JM. Novel model of frontal impact closed head injury in the rat. Journal of Neurotrauma. 2009;26: 2233-2243.

9. Giza CC Hovda, DA. The new neurometabolic cascade of concussion. Neurosurgery. 2014;75:4524-4533.

10. Duffy JR. Motor speech disorders. St. Louis MO, Mosby Pub 2013.

11. Murdoch BE, Kuruvilla MS, Goozee JV. Effect of speech rate manipulations on articulatory dynamics in severe traumatic brain injury: An EMA and EPG study. Brain Injury. 2012;26:241-260.

12. Toshniwal SS, Joshi NA. Residual speech impairment in patients with traumatic brain injury. Indian Journal of Neurotrauma. 2010; 7:61-66.

13. Vitorino J. Laryngeal function: a comparative analysis between children and adults subsequent to traumatic brain injury. Journal of Head Trauma Rehabilitation. 2009;24:374-383.

14. Ergun A, Oder W. Oral diadochokinesis and velocity of narrative speech: A prognostic parameter for the outcome of diffuse axonal injury in severe head trauma. Brain Injury. 2008;22:773-779.

15. Wang Y, Kent RD, Duffy JR, Thomas JE. Dysarthria associated with traumatic brain injury: speaking rate and emphatic stress. Journal of Communication Disorders. 2005:38:231-260.

16. Wang Y, Kent R, Duffy J, Thomas J, Weismer G. Alternating motion rate as an index of speech motor disorder in traumatic brain injury. Clinical Linguistics and Phonetics. 2004;18:57-84.

17. Cahill LM, Murdoch BE, Theodoros DG. Perceptual analysis of speech following traumatic brain injury in childhood. Brain Injury. 2002:16:415-446.

18. Ziegler W. Task-related in oral motor control: speech and oral diadochokinesis in dysarthria and apraxia of speech. Brain and Language. 2002;80:556-575.

19. Goozee JV, Murdoch BE, Theodoros DG. Physiological assessment of tongue function in dysarthria following traumatic brain injury. Logo Phon Voco. 2001;26:51-65.

20. Goozee JV, Murdoch BE, Theodoros DG, Stokes PD. Kinematic analysis of tongue movements in dysarthria following traumatic brain injury using electromagnetic articulography, Brain Injury. 2000;14:153-174.

21. Gadesmann M, Miller N. Reliability of speech diadochokinetic test measurement. International Journal of Language Communication Disorder. 2008;43:41-54.

22. Williams P, Stackhouse J. Rate, accuracy, and consistency: diadochokinetic performance of young, normally developing children. Clinical Linguistics and Phonetics.2000;14:267-293.

23. De Beaumont L, Theoret H, Mongeon D, Messier I, Leclerc S, Tremblay S, Ellemberg D, Lassonde M. Brain function decline in healthy retired athletes who sustained their last sports concussion in early adulthood. Brain: A Journal of Neurology. 2009;132:695708.

24. Elkin BE, Elloitt JM, Siegmun GP. Whiplash injuryor concussion? A possible biomechanical explanation concussion symptoms in some individuals following a rear-end collision. J Orthopaedic of sports physical therapy, 2016;46:874-884.

25. Kay Elemetrics CSL: Computerized Speech Laboratory, Model 4300B. 5.0 ed Lincoln Park NJ: Kay Elemetrics Corporation. 1994.

26. Kent RD, Reed C.The acoustic analysis of speech. 2nd Ed.Singualar/ Thomas Learing USA. 2002.

27. Cronk BC. How to Use SPSS: A step-by-step guide to analysis and interpretation. Glendale CA:Fred Pyrczak Pub. 2012. 